Trauma Berufskrankh 2008 10 [Suppl 1]:140-145

DOI 10.1007/s10039-007-1275-7

Online publiziert:30. August 2008

(c) Springer Medizin Verlag 2007

\author{
M. Militz \\ BG-Unfallklinik, Murnau
}

\title{
Strategien zur Eingrenzung multiresistenter Erreger im Krankenhaus
}

\author{
Konzept und Umsetzung einer Isoliereinheit
}

Wundinfektionen nach operativen Behandlungen stellen unverändert eine Herausforderung an die moderne Medizin dar. Neben der demografischen Entwicklung in den Industrienationen mit zunehmend älteren $\mathrm{Pa}$ tienten, welche durch die Fortschritte in allen Bereichen der medizinischen Versorgung trotz bestehender Multimorbidität auch elektive Eingriffe erfahren, ist der steigend breite Einsatz von Antibiotika in verschiedenen Bereichen Ursache für die zunehmende Resistenzentwicklung der Mikroorganismen. So war bereits kurze Zeit nach der Entdeckung des Penicillins und dessen industrieller Herstellung auch die Entwicklung von antibiotikaresistenten Bakterienstämmen bekannt.

Da die überwiegende Zahl der postoperativen Wundinfektionen in der Unfallchirurgie und Orthopädie von Staphylococcus aureus verursacht wird, sind die Entwicklung und Verbreitung des methicillinresistentenStaphylococcus aureus (MRSA) von herausragender Bedeutung.

Wesentlich für die Praxis sind:

- die Unempfindlichkeit von MRSA gegenüber einer Vielzahl herkömmlicher Antibiotika sowie

- die eingeschränkten Behandlungsoptionen ( $\bullet$ Abb. 1).

Staphylococcus-aureus-Infektion. Die Übertragung und Verbreitung von Staphylococcus aureus und damit auch von MRSA erfolgen v. a. über Tröpfchen-, Aerosol- und Kontaktübertragung bei ungenügenden basishygienischen Maßnahmen [6, 7, 14].

Bei konsequenter Umsetzung der Hygienemaßnahmen im Rahmen einer prospektiven Untersuchung konnte hinsichtlich der MRSA-Ansteckungsraten bzw. dem Typ der übertragenen Stämme auf einer Intensiveinheit kein signifikanter Unterschied in den Patientencharakteristika beobachtet werden [2]. Die Kernaussage dieser Arbeit bestand darin, dass bei einer entsprechenden Schulung des medizinischen Personals durch die Umsetzung der basishygienischen Maßnahmen eine Übertragung von Erregern innerhalb der Behandlungseinheiten nicht zu erwarten ist.

Häufigkeit und Risiken resistenter Erreger. Bemerkenswert ist, dass die Häufigkeit der multiresistenten Erreger mit dem Verbrauch von Antibiotika in einem engen Zusammenhang steht. Goossens et al. [4] konnten nachweisen, dass in Europa ein deutliches Süd-Nord-Gefälle hinsichtlich der Menge der verschriebenen Antibiotika besteht. Die gleichartige Verteilung der Resistenzraten in Süd- und Osteuropa wird im direkten Zusammenhang mit dem Antibiotikaverbrauch gesehen (• Abb. 2).

Es ist nicht verwunderlich, dass mit der Zunahme der multiresistenten Erreger, besonders des MRSA, die Anzahl der Wundinfektionen mit MRSA, besonders in den Ländern mit hoher MRSA-Dichte, ansteigt [1, 9, 12]. Nachvollziehbar ist, dass mit der zunehmenden Inzidenz multiresistenter Erreger auch eine Zunahme der Kosten für die Behandlung nosokomialer Infektionen mit multiresistenten Erregern resultiert $[8,10]$.

Des Weiteren konnte in zahlreichen Untersuchungen festgestellt werden, dass eine erhöhte Morbidität und Mortalität im direkten Zusammenhang mit der MRSAInzidenz stehen $[13,15,16,20]$.
Prävention. Es wurden zahlreiche Untersuchungen zur Prävention und Kontrolle nosokomialer Infektionen durch MRSA durchgeführt und publiziert:

- Es wird insbesondere die gezielte Beobachtung der MRSA-Träger empfohlen $[1,11,19]$.

- Die Isolierung als Maßnahme zur Reduktion der Übertragung von MRSA im Krankenhaus wird diskutiert $[1,3$, $11,18]$. Allerdings ist bisher nicht nachgewiesen, dass die Isolierung von MRSA-Trägern und Patienten mit MRSAWundinfektionen tatsächlich im Verhältnis zu dem dadurch erzielten Effekt der Verhinderung der Übertragung der MRSA auf andere Patienten steht. So wurde von Kappstein [7] und Neumaier et al. [14] dargelegt, dass die Umsetzung der Basishygienemaßnahmen wesentlich für die Verhinderung nosokomialer Infektionen auch beim MRSA ist. Nur Patienten mit einem hohen Risiko der Besiedelung mit MRSA sollten einer Screeninguntersuchung unterzogen werden. Sollte diese positiv ausfallen, ist eine Behandlung mit Mupirocin ausreichend. Unter Berücksichtigung des dargestellten Hygieneregimes wird das Übertragungsrisiko gering eingeschätzt.

- Die Erfahrungen aus den Niederlanden mit einer konsequenten ,surch und distroy"-Strategie hinsichtlich der Verhinderung der Übertragung von MRSA im Krankenhaus sind beeindruckend. So beträgt dort der Anteil klinischer Isolate von MRSA < $1 \%$ [17]. Neben der „surch und distroy“-Politik ist mit großer Wahrscheinlichkeit auch 
der restriktive Antibiotikaeinsatz wesentlich für die geringe Rate multiresistenter Erreger in den Niederlanden [4].

Problematisch in der praktischen Umsetzung wird die Isolierung von Patienten besonders unter psychischen Gesichtspunkten gesehen, da neben den beschränkten Bewegungsmöglichkeiten für die Patienten auch eine Stigmatisierung befürchtet wird. Als wesentlich für eine Verminderung der psychischen Belastung werden die konsequente Einhaltung und Umsetzung des Hygieneregimes von allen Mitarbeitern gesehen, um die Akzeptanz und das Vertrauen in die erforderlichen Maßnahmen zu stärken [5].

Vor diesem Hintergrund wurde in der BG-Unfallklinik Murnau im Jahr 2004 der Entschluss gefasst, eine Station für die multiresistenten Erreger einzurichten. Ziel einer solchen ist es, die Patienten mit multiresistenten Erregern zu isolieren, um die Möglichkeiten des Kontakts der MRSATräger mit anderen Patienten und Personal und damit eine potenzielle Übertragung zu reduzieren. Durch eine technisch hochwertige Ausstattung sollte der Umfang der Patiententransporte auf ein Mindestmaß reduziert werden, ohne die medizinische Versorgung der Patienten zu beeinträchtigen.

\section{Praktische Umsetzung}

Seit November 2005 konnte die so genannte MRE-Station (Multiresistente-ErregerStation) an der BG-Unfallklinik Murnau mit 12 Betten in Betrieb genommen werden. Dabei handelt es sich um Einzelzimmer, die im Bedarfsfall auch als Zweitbettzimmer eingerichtet werden können. Jedes Zimmer ist mit einer komfortablen Nasszelle und einem Terrassenausgang ausgestattet. Außerdem ist jedes Zimmer einzeln über eine entsprechende Schleuse zu erreichen. Mit dieser räumlichen Ausstattung können die Maßnahmen

- des Screenings,

- der räumlichen Trennung der MRSAPatienten,

- derEradikationvonMRSA-Trägernund

- des konsequenten Hygienemanagements

umgesetzt werden.

Trauma Berufskrankh 2008 · 10[Suppl 1]:140-145 DOI 10.1007/s10039-007-1275-7

(c) Springer Medizin Verlag 2007

M. Militz

Strategien zur Eingrenzung multiresistenter Erreger im
Krankenhaus. Konzept und Umsetzung einer Isoliereinheit

\section{Zusammenfassung}

Der steigend breite Einsatz von Antibiotika ist für die zunehmende Resistenzentwicklung der Mikroorganismen verantwortlich. In der Unfallchirurgie und Orthopädie wird die überwiegende Zahl der postoperativen Wundinfektionen durch Staphylococcus aureus verursacht, weshalb die Entwicklung und Verbreitung des methicillinresistentenStaphylococcus aureus (MRSA) von herausragender Bedeutung sind. Bei der Behandlung von MRSA-Infektionen sind insbesondere die Unempfindlichkeit von MRSA gegenüber einer Vielzahl herkömmlicher Antibiotika sowie die eingeschränkten Behandlungsoptionen von Bedeutung. Somit kommt der Prävention der Übertragung und damit basishygienischen Maßnahmen eine überragende Bedeutung zu. Die Einrichtung von
Isolierstationen könnte hierfür eine Option sein. Anhand der Isolierstation der BGUnfallklinik Murnau wird aufgezeigt, wie eine solche Station konzipiert sein sollte, welche Therapie- und Hygienemaßnahmen erforderlich werden und wie die Versorgungsqualität gewährleistet werden kann. Die Isolierung potenziell infektiöser Patienten hat zu einer Reduktion von MRSA-Infektionen geführt, gleichzeitig haben das Verständnis für die MRSA-Problematik und das Bewusstsein für die basishygienischen Maßnahmen zugenommen.

\section{Schlüsselwörter}

Staphylococcus aureus · Isolierung · Screening $\cdot$ Resistenzentwicklung $\cdot$ MSRA

\section{Strategies for restricting the spread of multiresistant pathogens in hospitals. Design and implementation of an isolation unit}

\section{Abstract}

The increasingly wide use of antibiotics is responsible for the increasingly frequent development of resistant microorganisms. In trauma and orthopaedic departments the vast majority of postoperative wound infections are caused by Staphylococcus aureus, which means the development and dissemination of methicillin-resistant Staphylococcus aureus (MRSA) is extremely significant. In the treatment of MRSA infections its insensitivity to a large number of conventional antibiotics and the restricted treatment options are importan factors. Extreme significance therefore attaches to prevention of its transmission, and thus to basic hygiene measures. Setting up isolation wards could be one option for this purpose. The isolation ward in the employers' liability insurance association's trauma clinic in Murnau is used as an example to show how a ward of this kind should be designed, what treatment and hygiene methods are required and how the quality of care can be guaranteed. The isolation of potentially infectious patients has led to a reduction in the number of MRSA infections, while at the same time understanding of the problems surrounding MRSA and awareness about the basic hygiene measures have improved.

\section{Keywords}

Staphylococcus aureus · Isolation · Screening $\cdot$ Resistant microorganisms · MRSA 

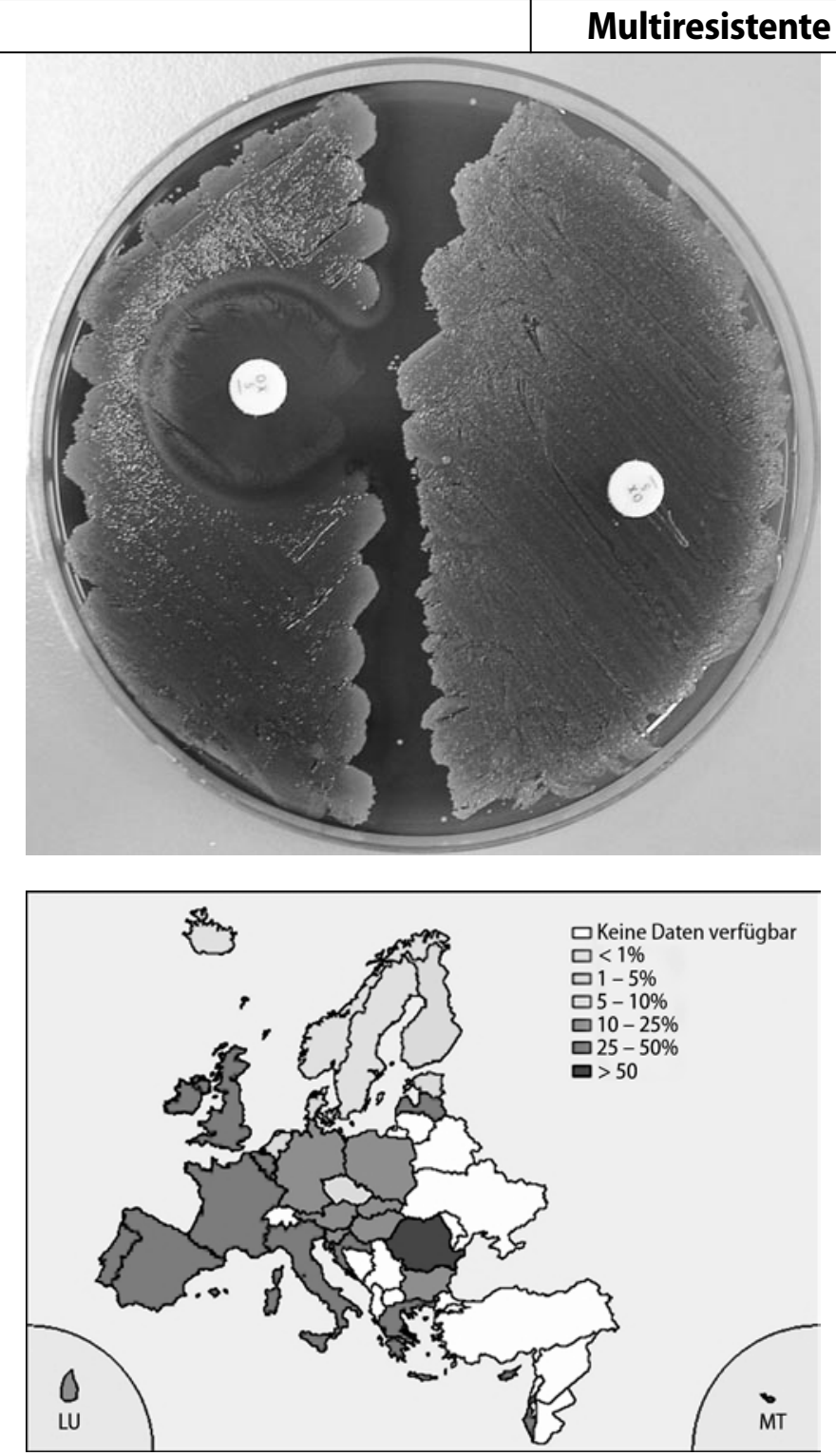

Um Hochrisikopatienten mit einer möglichen MRSA-Besiedelung oder Infektion detektieren zu können, werden die Patienten, die ein hohes Risiko aufgrund eines Aufenthalts in einem ausländischen Krankenhaus, in einem Altenheim bzw. mit bekannter MRSA-Anamnese haben, einem Screening des Nasen-Rachen-Raums, der Leiste und der Wunde sowie bei rückenmarkverletzten Patienten auch des Urins unterzogen. Nach Vorliegen der Abstrichergebnisse, frühestens nach $24 \mathrm{~h}$, können eine Entisolierung bzw. eine weitere Isolierung der Patienten veranlasst werden (• Abb. 3).

\section{Infobox 1: Internetlinks}

- MRSA-Inzidenz in Europa 2004: http:// www.bioquell.com/us/microbiology. asp?id=141
Abb. $2<$ MRSA-Inzidenz in Europa 2004 (Quelle: D s. Infobox 1)

Abb. $1<$ Bluta-

garschälchen mit oxacillinsensiblem Staphylococcus aureus links und oxacillinresistentem Staphylococcus aureus (ORSA) rechts (keine Hemmung des Bakterienwachstums plättchen rechts)

\section{Ergebnisse}

\section{Patienten und Infektionen}

2006 wurden insgesamt 434 Patienten auf der MRE-Station behandelt, davon 170 aus der eigenen Klinik, 264 aus anderen Krankenhäusern. Nosokomiale Übertragungen wurden bei 98 Patienten beobachtet, davon 77 bei Patienten aus anderen Krankenhäusern. Die 21 Übertragungen in der BG-Unfallklinik Murnau waren zu knapp 50\% auf der Intensivstation zu verzeichnen, gefolgt von den Stationen der Rückenmarkverletztenabteilung. Auf der MRE-Station kam es lediglich in 1 Fall zu einer Übertragung.

Der Anteil der MRSA-Patienten auf der Station beträgt durchschnittlich etwa $50 \%$, sodass aufgrund der Konzeption der durch das Oxacillin-
Station auch Patienten ohne Isolierungsmaßnahmen behandelt werden.

Die Screeningmaßnahmen waren zunächst auf ein breites Patientenklientel ausgedehnt. Im Verlauf des Jahres 2006 wurde jedoch evident, dass nur etwa 10\% der Patienten, die in diesem Risikoprofil gescreent wurden, einen positiven MRSA-Nachweis aufwiesen. Aus diesem Grund wurde das Risikoprofil für die Screeninguntersuchung Mitte 2006 modifiziert. Dadurch kam es zu einer deutlichen Verminderung der Screeninguntersuchungen mit einer prozentualen Erhöhung der positiven MRSA-Nachweise (• Abb. 4).

\section{Pflegetage auf der MSRA-Station}

Seit Mitte 2006 waren die räumlichen und personellen Voraussetzungen auch für die Behandlung von rückenmarkverletzten Patienten mit MRSA-Besiedelung erfüllt, sodass ab diesem Zeitpunkt eine deutliche Zunahme der Pflegetage mit MRSA auf der MRE-Station beobachtet werden konnte (• Abb.5).

\section{Therapie- und Hygienemaßnahmen}

Um eine Eradikation des MRSA zu erreichen, werden desinfizierende Ganzkörperwaschungen sowie die Behandlung mit Mupirocinnasensalbe bei den Patienten ohne offene Wunden durchgeführt. Liegen im weiteren Verlauf an 3 aufeinander folgenden Tagen negative Abstriche vor, wird eine Entisolierung des Patienten vorgenommen. Im weiteren Verlauf werden weiterhin die Haut- und Nasenbesiedelung kontrolliert, um ein erneutes Auftreten der MRSA-Kontamination verfolgen zu können.

Wesentlicher Bestandteil der Hygienemaßnahmen ist die konsequente Umsetzung der Basishygienemaßnahmen und des Monitorings. So konnte im Rahmen der regelmäßig durchgeführten Abstriche von den Einrichtungsgegenständen in den Isolierzimmern keine Besiedelung mit MRSA festgestellt werden.

\section{Psychische Belastung, Versorgungsqualität}

Aus den Angaben der Literatur [5, 7] ist bekannt, dass Isolierungsmaßnahmen ei- 

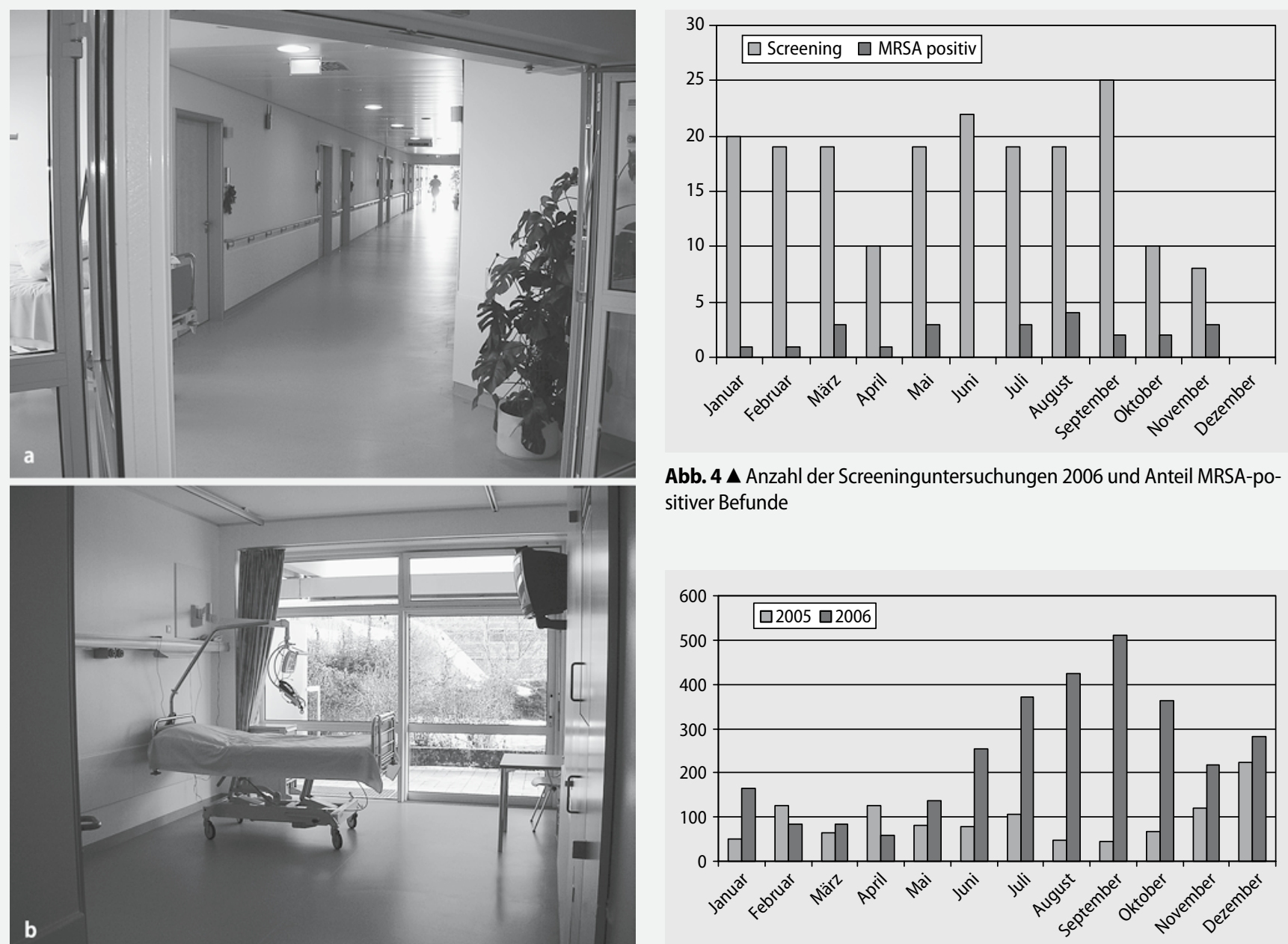

Abb. 4 A Anzahl der Screeninguntersuchungen 2006 und Anteil MRSA-positiver Befunde
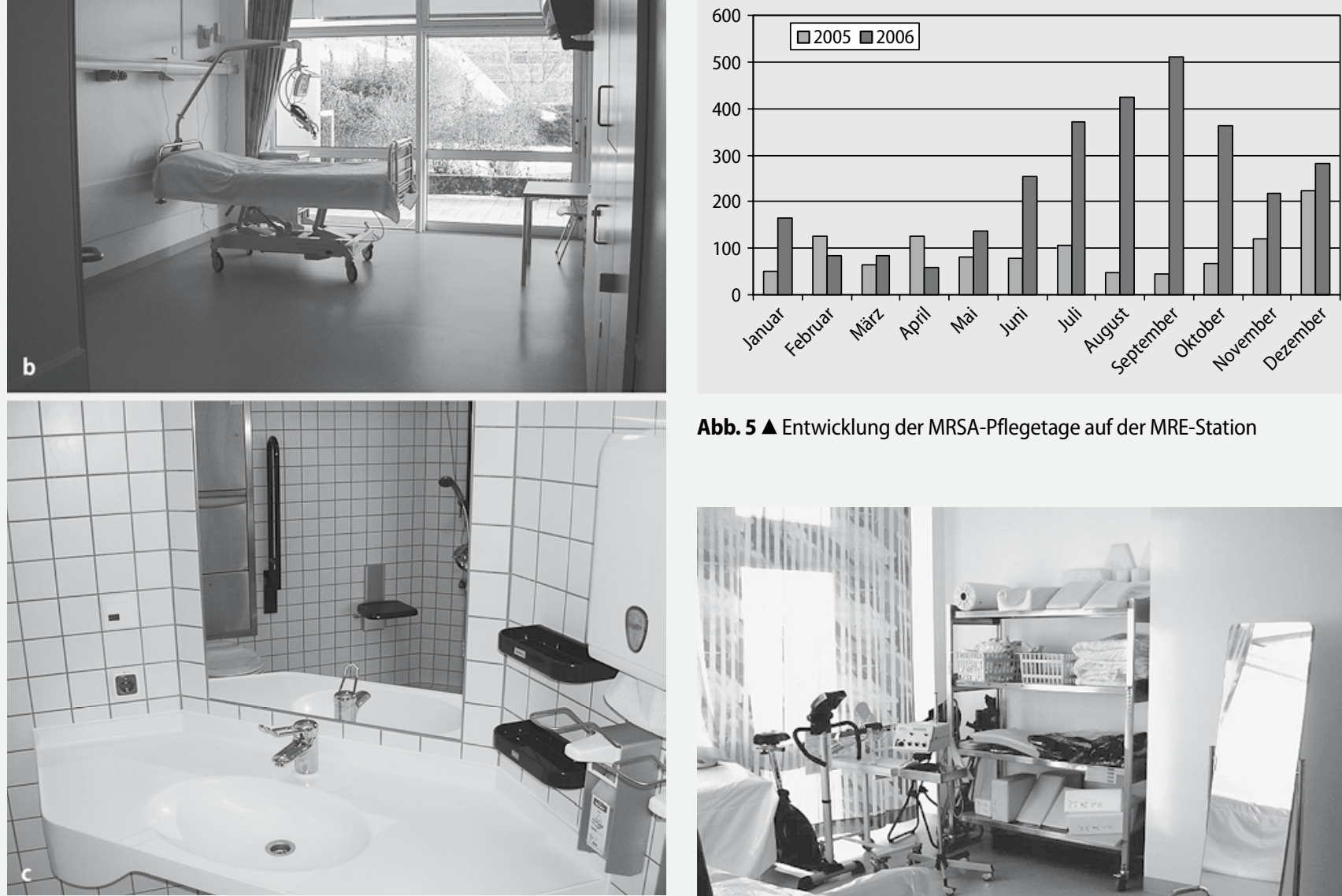

Abb. $3 \Delta$ MRE-Station der BG-Unfallklinik Murnau, a MRE-Station mit 12 Einzelzimmern, b Patientenzimmer mit Terrassenzugang und c Nasszelle

Abb. 5 A Entwicklung der MRSA-Pflegetage auf der MRE-Station

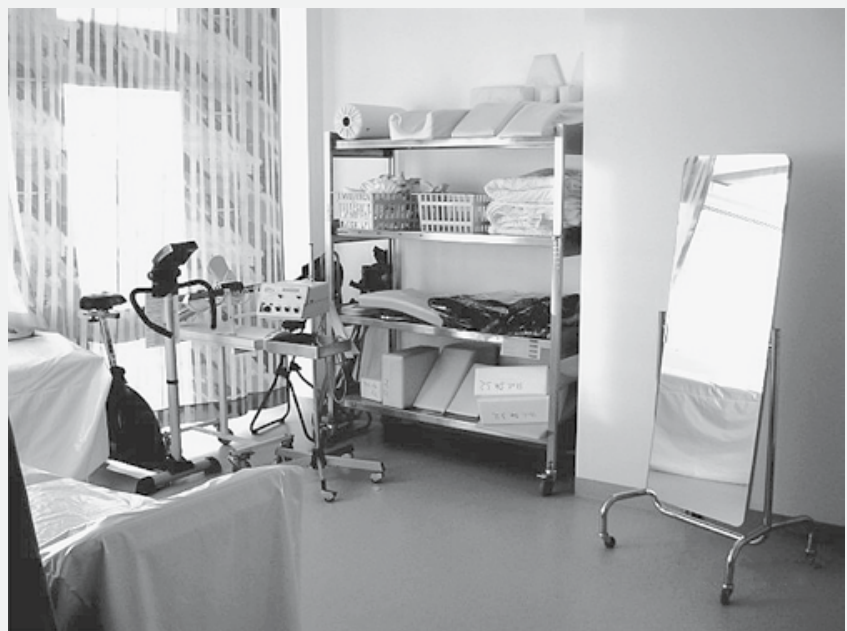

Abb. $6 \Delta$ Separate Geräte für Krankengymnastik, Ergotherapie und Lagerungsmittel 


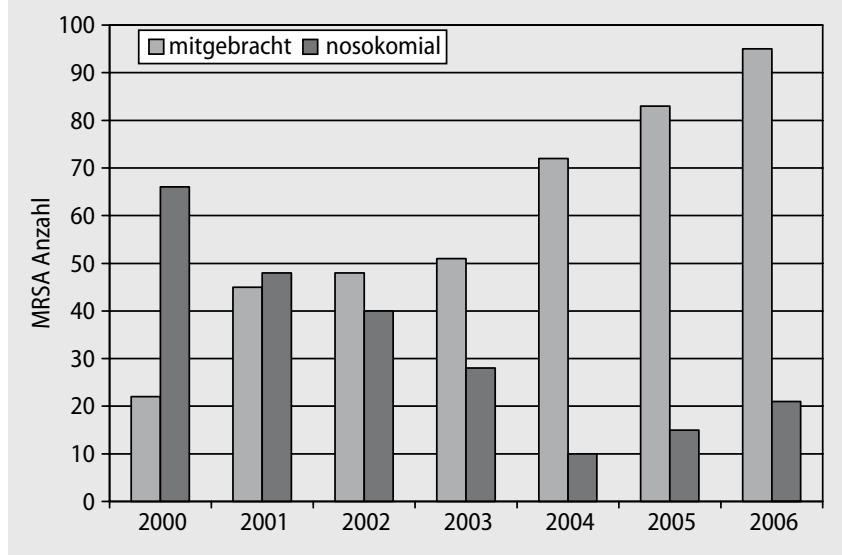

kritischem Umgang mit dieser Frage werden diese Maßnahmen auch von den Patienten akzeptiert und mitgetragen.

\section{Schlussfolgerung}

Wir haben in den letzten $11 / 2$ Jahren die
Abb. $7 \varangle$ Entwicklung der nosokomialen und auswärtigen MRSAPatienten in der BGUnfallklinik Murnau 2000-2006 ne erhöhte psychische Belastung der Patienten darstellen. Auch eine Verschlechterung der medizinischen Versorgung im Zusammenhang mit Isolierungsmaßnahmen wird diskutiert.

Zur Prävention einer derartigen Entwicklung und Reduktion der Patientenund Personalbewegungen wurden eine separate Krankengymnastik- und Ergotherapieausrüstung angeschafft (- Abb. 6). Außerdem befinden sich auf der Station ein EKGund Ultraschallgerät sowie ein Röntgengerät zur Durchführung von Thoraxaufnahmen bei Patienten mit Rückenmarkverletzungen und Tracheostoma. Damit werden die Fluktuation der Patienten mitMRSA-Besiedelung und die Personalbewegungen im Krankenhaus reduziert, die medizinische Versorgung jedoch gewährleistet.

Wegen der potenziellen psychischen Belastung durch die Isolationsmaßnahmen wurde ein Informationsblatt für die Patienten und Besucher erarbeitet. Diese Informationen werden dem Patienten mit Beginn der Behandlung ausgehändigt. Sollte dennoch aufgrund der Erkrankung und der zusätzlichen Isolationsmaßnahmen ein Bedarf an einer psychischen Betreuung bestehen, kann diese jederzeit realisiert werden.

\section{Diskussion}

Die steigende Zahl nosokomialer Infektionen mit MRSA zwingt aufgrund der eingeschränkten Behandlungsmöglichkeiten mit Antibiotika zu einer konsequenten Strategie beim Umgang mit multiresistenten Erregern. Trotz der unterschiedlichen Erfahrungen und Aussagen in der Literatur wurde in der BG-Unfallklinik Murnau eine interdisziplinäre MRE-Station eingerichtet. Die ersten Erfahrungen dieser in- terdisziplinären Isoliereinheit zeigen, dass trotz der Isolierungsmaßnahmen, die eine nosokomiale Übertragung weitgehend verhindern konnten, die medizinische Versorgung der Patienten mit nur geringen Einschränkungen realisiert werden kann.

Nach unseren Erfahrungen wird durch die gezielten Screeninguntersuchungen eine frühzeitige Erkennung von Patienten mit MRSA-Wundinfektionen bzw. von MRSA-Trägern erreicht. Bei erfolgreicher Eradikation ist eine Verlegung der Patienten auf die Station der entsprechenden

Wesentlich für den Erfolg der Isoliereinheit sind:

- das konsequente Hygienemanage-
ment und
- der restriktive Antibiotikaeinsatz.

Die Entwicklung der MRSA-Inzidenz in der BG-Unfallklinik Murnau in den letzten Jahren zeigt, dass die bisherigen Strategien zur Kontrolle und Prävention der MRSA-Infektion und -Besiedelung erfolgreich sind. Die Anzahl der nosokomialen Infektionen, die in der BG-Unfallklinik Murnau erworben wurden, ist in den letzten Jahren kontinuierlich zurückgegangen, wohingegen die Anzahl der zuverlegten Patienten kontinuierlich steigt (• Abb. 7). Die Umsetzung einer solchen Isoliereinheit bedeutet ein hohes Engagement der Mitarbeiter in allen Ebenen, um den Herausforderungen der physischen und psychischen Belastungen durch die Isolierungsmaßnahmen und der Umsetzung der Hygienemaßnahmen gewachsen zu sein.

Nach unserer Einschätzung wird durch die Screening- und Isolierungsmaßnahmen das Bewusstsein für die basishygienischen Maßnahmen geschärft. Bei offenem und Fachabteilungen möglich. Erfahrung gemacht, dass durch die Einrichtung der Isoliereinheit das Verständnis für die MRSA-Problematik, sowohl beim Pflegepersonal als auch beim ärztlichen Personal, zugenommen hat. Durch die Einrichtung von Standards und ein Hygienemonitoring kann der Nachweis der Effektivität der Maßnahmen erbracht werden. Negative Effekte durch die Isolierungsmaßnahmen bei den betroffenen Patienten konnten unsererseits bisher nicht beobachtet werden.

\section{Korrespondenzadresse}

Dr. M. Militz

BG-Unfallklinik Murnau,

Prof.-Küntscher-Straße 8, 82418 Murnau

mmilitz@bgu-murnau.de

Interessenkonflikt. Der korrespondierende Autor gibt an, dass kein Interessenkonflikt besteht.

\section{Literatur}

1. Arnold MS, Dempsey JM, Fishman M et al. (2002) The best hospital practices for controlling methicillin-resistant Staphylococcus aureus: on the cutting edge. Infect Control Hosp Epidemiol 23: 69-76

2. Cepeda JA, Whitehouse T, Cooper B et al. (2005) Isolation of patients in single rooms or cohorts to reduce spread of MRSA in intensive-care units: prospective two-centre study. Lancet 365: 295-304

3. Coia JE, Duckworth GJ, Edwards DI et al. (2006) Guidelines for the control and prevention of meticillinresistant Staphylococcus aureus (MRSA) in healthcare facilities. J Hosp Infect [Suppl 1] 63: S1-44

4. Goossens H, Ferech M, Vander SR et al. (2005) Outpatient antibiotic use in Europe and association with resistance: a cross-national database study. Lancet 365: 579-587

5. Hartmann C (2005) Wie erleben Patienten die Isolierung wegen einer Infektion oder Kolonisierung mit MRSA? Hyg Med 7/8: 234-243

6. Kappstein I (2006) [Current issues of methicillin-resistant Staphylococcus aureus: epidemiology, diagnostics, prevention, and therapy]. Chirurg 77: 499-505

7. Kappstein I (2006) Prävention von MRSA-Übertragungen. Krankenhaushygiene up2date 1:117-130

8. Kim T, Oh PI, Simor AE (2001) The economic impact of methicillin-resistant Staphylococcus aureus in Canadian hospitals. Infect Control Hosp Epidemiol 22: 99-104

9. Kola A, Chaberny IF, Mattner F et al. (2006) [Control of methicillin-resistant $S$. aureus by active surveillance. Results of a workshop held by the Deutsche Gesellschaft fur Hygiene und Mikrobiologie]. Anaesthesist 55: 778-783 
10. Kreutzer J, Nussbaum B, Bernd L et al. (2002) Reale Kosten der MRSA-Osteitis. Trauma Berufskrankh 4: 344-349

11. Loveday HP, Pellowe CM, Jones SR et al. (2006) A systematic review of the evidence for interventions for the prevention and control of methicillinresistant Staphylococcus aureus (1996-2004): report to the Joint MRSA Working Party (Subgroup A). J Hosp Infect [Suppl 1] 63: S45-S70

12. Manian FA, Senkel D, Zack J et al. (2002) Routine screening for methicillin-resistant Staphylococcus aureus among patients newly admitted to an acute rehabilitation unit. Infect Control Hosp Epidemiol 23: 516-519

13. Minary-Dohen P, Bailly P, Bertrand X et al. (2003) Methicillin-resistant Staphylococcus aureus (MR$\mathrm{SA})$ in rehabilitation and chronic-care-facilities: what is the best strategy? BMC Geriatr 3:5

14. Neumaier M, Kappstein I, Scherer MA (2006) [Positive screening for MRSA - clinical consequences?]. Unfallchirurg 109: 499-504

15. Nixon M, Jackson B, Varghese P et al. (2006) Methicillin-resistant Staphylococcus aureus on orthopaedic wards: incidence, spread, mortality, cost and control. J Bone Joint Surg Br 88: 812-817

16. Shorr AF, Tabak YP, Gupta V et al. (2006) Morbidity and cost burden of methicillin-resistant Staphylococcus aureus in early onset ventilator-associated pneumonia. Crit Care 10: R97

17. Vriens MR, Blok HE, Gigengack-Baars AC et al. (2005) Methicillin-resistant Staphylococcus aureus carriage among patients after hospital discharge. Infect Control Hosp Epidemiol 26: 629-633

18. Wertheim HF, Vos MC, Boelens HA et al. (2004) Low prevalence of methicillin-resistant Staphylococcus aureus (MRSA) at hospital admission in the Netherlands: the value of search and destroy and restrictive antibiotic use. J Hosp Infect 56: 321-325

19. West TE, Guerry C, Hiott M et al. (2006) Effect of targeted surveillance for control of methicillin-resistant Staphylococcus aureus in a community hospital system. Infect Control Hosp Epidemiol 27: 233-238

20. Wyllie DH, Crook DW, Peto TE (2006) Mortality after Staphylococcus aureus bacteraemia in two hospitals in Oxfordshire, 1997-2003: cohort study. BMJ 333: 28 